\title{
Conditionnel et ultériorité dans le PASSÉ : de la subjectivité à l'objectivité
}

\author{
Jacques Bres \\ Praxiling UMR 5267, CNRS-Montpellier III
}

\begin{abstract}
Comment signifier qu'un procès $\mathrm{B}$, est, dans le PASSÉ $^{1}$ ultérieur à un procès $\mathrm{A}^{2}$ ? Le français, comme les autres langues romanes, s'est doté, sur le modèle des formes du FUTUR, de formes aptes à exprimer l'ultériorité dans le PASSÉ subjectivement (point 1). Pas seulement cependant : il a également réussi à développer l'expression de l'ultériorité objective dans le PASSÉ (point 2), par un chemin de grammaticalisation que nous essayons de tracer pour le conditionnel (point 3.).
\end{abstract}

\section{Antériorité, ultériorité}

On peut considérer :

- les temps du PASSÉ (passé simple, imparfait, passé composé, passé immédiat) ${ }^{3}$ comme permettant de signifier l'antériorité à partir du PRÉSENT. Dire :

(1) Corinne vint / venait / est venue /vient de venir me voir,

c'est poser déictiquement l'acte de venir comme ayant eu lieu dans l'antériorité du nunc de l'énonciation ${ }^{4}$.

- les formes du FUTUR (futurs synthétique et périphrastiques) comme permettant de signifier l'ultériorité à partir du PRÉSENT. Dire :

(2) Corinne viendra / va venir / doit venir me voir,

c'est déictiquement prévoir l'acte de venir comme devant/pouvant avoir lieu dans l'ultériorité du nunc de l'énonciation. Ultériorité qui, à la différence de l'antériorité, a affaire au champ des possibles si l'on considère, à la suite des logiques du temps, que le FUTUR est ramifié, ce qui n'est le cas ni du PASSÉ ni du PRÉSENT qui sont unilinéaires (Gardies 1975, Martin 1981, Vuillaume 2001) : quelque certitude, désir (ou crainte) que je puisse avoir que Corinne vienne demain, dans les faits elle pourra venir ou ne pas venir (pour le lien futur / modalité épistémique, cf. inter alia, Bybee et al.1994 : 83, Tournadre 2004 : 57); alors que si je dis :

(1a) Corinne est venue me voir hier,

l'acte de venir - sauf énonciation mensongère - est de l'ordre de l'effectif et non du possible.

\begin{tabular}{|c|c|c|}
\hline & PASSÉ & FUTUR \\
\hline antériorité & vint, venait, est venue, vient de venir & $\varnothing$ \\
\hline ultériorité & $\varnothing$ & viendra, va venir, doit venir \\
\hline
\end{tabular}

Tableau 1

Cette représentation est cependant très incomplète. Les constructeurs de langue (Hagège, 1993) ont ressenti le besoin discursif de signifier également l'ultériorité dans le PASSÉ, et ont usé pour cela des structures employées dans l'expression de l'ultériorité du PRÉSENT : - $r$-d'ultériorité pour la forme synthétique, aller et devoir pour les formes périphrastiques, mais combinées avec un morphème de PASSÉ, pour le français celui de l'imparfait : 
(3) Corinne m'a dit qu'elle viendrait / allait/ devait venir me voir.

On a donc, pour signifier l'ultériorité dans le PASSÉ, sur le modèle des formes de l'ultériorité du PRÉSENT, le conditionnel viendrait, et les deux formes périphrastiques allait / devait venir formées sur l'imparfait des auxiliaires aller et devoir. Soit donc, en complément du précédent tableau :

\begin{tabular}{|c|c|c|}
\hline & PASSÉ & FUTUR \\
\hline antériorité & vint, venait, est venue, vient de venir & $\emptyset$ \\
\hline ultériorité & viendrait, allait venir, devait venir & viendra, va venir, doit venir \\
\hline
\end{tabular}

Tableau 2

Les grammairiens ont de longue date noté la symétrie dans l'expression de l'ultériorité du PRÉSENT et de l'ultériorité dans le PASSÉ, qui se manifeste notamment dans la concordance des temps :

(4) Corinne me dit qu'elle viendra me voir

(3a) Corinne m'a dit qu'elle viendrait me voir.

Et parlent du conditionnel comme d'un « futur dans le passé » ${ }^{5}$ : si l'expression de futur est malheureuse il nous semble plus juste de parler d'ultérieur -, elle pointe ce parallélisme. Moins d'attention a été prêtée à un fait énonciatif lourd de conséquences : alors que le futur, en tant qu'ultérieur du PRÉSENT, se construit à partir du nunc de deux façons: directement ((2) Corinne viendra me voir) ou via une énonciation rapportée simultanée ((4) Corinne me dit qu'elle viendra me voir), le conditionnel «temps » ne peut se construire directement: l'énoncé Corinne viendrait me voir n'actualise le sens temporel d'ultériorité que dans le cadre syntactico-énonciatif du discours rapporté indirect (3a), ou indirect libre (3b) :

(3b) Je souriais : oui, Corinne viendrait me voir.

Soit donc :

- avec le futur, l'ultériorité s'organise déictiquement à partir de l'acte d'énonciation (E) du locuteurénonciateur $\mathrm{E}_{1}$. Le procès est posé dans le $\mathrm{FUTUR}^{6}$;

- avec le conditionnel, l'ultériorité s'organise à partir d'un acte d'énonciation antérieur (e) réalisé par un

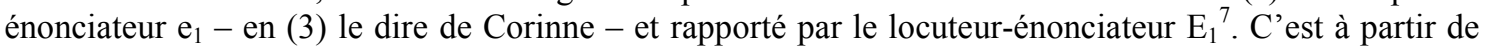
cette instance (qui n'est pas un simple point de repère, comme chez Reichenbach, 1947/1966) que le procès est construit en ultériorité anaphoriquement, ce qui rend compte de ce qu'il puisse référer à un événement antérieur, contemporain ou postérieur au $n u n c^{8}$.

Cette différence se manifeste par le fait suivant: l'ultériorité signifiée par le futur peut être précisée seulement par un circonstant référant au FUTUR de (E); l'ultériorité signifiée par le conditionnel peut être précisée par un circonstant pouvant correspondre au PASSÉ, au PRÉSENT comme au FUTUR de (E) :

\section{(4a) Corinne (me dit qu'elle) viendra demain (aujourd'hui $\left.{ }^{9}\right) /$ hier $^{10}$ \\ (3c) Corinne m'avait dit qu'elle viendrait me voir la semaine dernière / aujourd'hui / la semaine prochaine.}

La structure temporelle du conditionnel en tant qu'ultérieur du PASSÉ est donc à l'origine d'un fonctionnement énonciatif spécifique. Nous avons avancé dans des travaux antérieurs que le conditionnel était un temps dialogique en langue en ce que, du fait de l'interaction des deux affixes - $r$ - et -ai qui le composent, il double systématiquement l'énonciation principale (E) d'une énonciation rapportée (e) antérieure, explicite (discours indirect) ou implicite (discours indirect libre). 
Ce que nous venons de développer pour le conditionnel en tant qu'ultérieur du PASSÉ subjectif, vaut, mutatis mutandis, pour les formes périphrastiques en allait et devait, avec lesquelles il alterne parfois, comme dans les occurrences suivantes :

(6) Alors il eut une envie immense de fuir (...) loin de cette passion furieuse qui le ravageait. Il allait retourner à Chatou, prendre le train, et ne reviendrait plus, ne la reverrait plus jamais. (Maupassant, La Femme de Paul)

(7) c'était le mois prochain qu'ils devaient s'enfuir. Elle partirait d'Yonville comme pour aller faire des commissions à Rouen. (Flaubert, Mme Bovary)

En (6), l'ultériorité du procès retourner, induite par l'auxiliaire allait, s'origine dans la pensée du personnage, son « envie immense de fuir »; en (7), l'ultériorité du procès s'enfuir, induite par l'auxiliaire devait, présuppose qu'il y a eu une interaction verbale antérieure - donc un acte d'énonciation - au cours de laquelle a été programmé comme ultérieur l'acte de s'enfuir, qui s'origine de plus ici dans la rêverie d'Emma. Les formes périphrastiques peuvent être remplacées, en grossière équivalence, par le conditionnel :

(6a) Il allait retourner à Chatou $\approx$ il retournerait à Chatou

(7a) c'était le mois prochain qu'ils devaient s'enfuir $\approx$ s'enfuiraient

Conclusion: le français, comme beaucoup d'autres langues, notamment les autres idiomes romans, a développé, parallèlement à l'expression verbo-temporelle de l'ultériorité du PRÉSENT, une expression verbo-temporelle de l'ultériorité dans le PASSÉ, en appui, dans les deux cas, sur une énonciation : celle $\mathrm{du}$ locuteur-énonciateur $\mathrm{E}_{1}$ pour le futur (et pour les tours périphrastiques en aller et devoir); celle, antérieure et rapportée, d'un énonciateur $\mathrm{e}_{1}$, pour le conditionnel (et pour les tours périphrastiques en aller et devoir). On a donc, dans les deux cas, une ultériorité subjective ${ }^{11}$, déictique pour le futur, et anaphorique pour le conditionnel.

Les différences entre expression de l'ultériorité dans le PASSÉ et expression de l'ultériorité du PRÉSENT ne s'arrêtent pas là : si l'ultériorité du PRÉSENT est toujours subjective, le français est parvenu à développer une ultériorité objective dans le PASSÉ - c'est-à-dire qui ne passe pas par la médiation d'un acte d'énonciation rapporté - à partir des formes verbo-temporelles de cette époque allait / devait + inf., conditionnel - mais également à partir du futur (dit dans cet emploi historique) ${ }^{12}$ (cf. infra: (11 -(14)), ce que nous allons analyser. Soit donc un troisième tableau :

\begin{tabular}{|c|c|c|}
\hline & PASSÉ & FUTUR \\
\hline antériorité & vint, venait, est venue, vient de venir & $\varnothing$ \\
\hline ultériorité subjective & viendrait, allait venir, devait venir & viendra, va venir, doit venir \\
\hline ultériorité objective & $\begin{array}{c}\text { viendrait, allait venir, devait venir, } \\
\text { viendra }\end{array}$ & $\varnothing$ \\
\hline
\end{tabular}

Tableau 3

\section{De l'ultériorité subjective à l'ultériorité objective dans le PASSÉ}

\subsection{Implicite de la progression temporelle adjacente et marquage par un circonstant de la progression temporelle non adjacente dans le PASSÉ}

Comment fait-on en français pour signifier objectivement la progression temporelle entre deux procès adjacents, à savoir dont la borne terminale du premier est séparée de la borne initiale du second par un espace de temps minimal?

(8) Ils se couchèrent. Elle souffla la bougie; et tout le monde bientôt dormit aux deux étages de la maison. (Maupassant, En famille) 
Soit la progression se couchèrent $<$ souffla $<$ s'endormit. Ces trois procès réfèrent à des actes que, de par nos connaissances du monde, nous comprenons comme successifs adjacents : la progression temporelle ne se marque pas (se couchèrent < souffla), notamment entre deux procès d'aspect lexical réalisation instantanée; ou se marque par un adverbe de succession immédiate comme bientôt (« tout le monde bientôt dormit »), notamment devant un procès d'aspect lexical activité comme dormir ${ }^{13}$.

Lorsque les deux procès sont distants temporellement, un circonstant temporel de temps ou de datation (antéposé le plus souvent au second procès) tend à marquer la progression :

(9) quand elle sceut la vérité, feit chercher le Cordelier partout, mais il estoit bien loing; et oncques puis ne fut trouvé en royaulme de France. (Marguerite de Navarre, Heptaméron)

(10) Le même jour, un incendie se manifesta dans la ville ; les Buonaparte virent leur maison brûler; quelque temps après, un décret les condamna à un bannissement perpétuel. (Dumas, Napoléon)

La progression entre les deux procès quelque peu distants temporellement s'appuie sur oncques puis en (9), sur quelque temps après en (10).

Notons que dans ces occurrences, la progression est objective : elle est posée par le narrateur, sans médiation énonciative d'un personnage.

\subsection{Du marquage par un circonstant de la progression temporelle objective au marquage de l'ultériorité objective par le verbe}

Jusqu'au XVIII ${ }^{\mathrm{e}} \mathrm{s}$ - et c'est encore le cas le plus fréquent de nos jours - la progression temporelle objective entre deux procès distants se signifie par des circonstants, et / ou est inférée contextuellement, mais elle n'est pas marquée sur le second procès lui-même.

On dispose donc, pour le PASSÉ :

- de circonstants pour marquer la progression temporelle objective entre deux procès distants (occurrences (9), (10)) ;

- de formes verbales pour signifier l'ultériorité subjective d'un procès à partir d'une énonciation rapportée (e) : conditionnel, allait / devait + inf. (occurrences (6), (7)).

Autour des $\mathrm{XVIII}^{\mathrm{e}}-\mathrm{XIX}^{\mathrm{e}} \mathrm{s}$, on assiste à l'innovation suivante : la langue réussit à signifier la progression temporelle objective entre deux procès distants à partir des outils de l'ultériorité subjective, à savoir le temps verbal du second procès (avec l'aide fréquente mais pas systématique d'un circonstant). Ce qui entraîne un léger changement sémantique : l'effet de sens produit n'est plus la simple progression temporelle entre deux procès éloignés dans le temps, mais la mise en perspective d'ultériorité objective du second procès à partir du premier procès (infra 3). Soit donc, pour l'ex. (10), et en remplacement du passé simple condamna:

\section{(10a) quelque temps après, un décret allait/devait les condamner/les} condamnerait/les condamnera à un bannissement perpétuel.

Proposons, pour chaque tour, une occurrence authentique de mise en perspective de la progression temporelle comme ultériorité objective (dorénavant on parlera par simplification d'ultériorité objective) :

(11) La seconde représentation, le 12, eut lieu devant une salle à moitié vide ; deux jours plus tard, moins de monde encore. La chute de Benvenuto allait faire renoncer Berlioz au théâtre pendant vingt-cinq ans. (Dumesnil, Histoire illustrée du théâtre lyrique, 1953)

(12) Toute cette pauvre famille proscrite s'embarqua sur un frêle bâtiment, et le futur César mit à la voile, protégeant de sa fortune ses quatre frères, dont trois devaient être rois, et ses trois sœurs, dont l'une devait être reine. (Dumas, Napoléon, 1839) 
(13) L'été 43 chassa la petite famille de la maison aux toits d'ardoise. Beaucoup plus tard, les enfants regretteraient les cerisiers, les buissons drus où ils enfouissaient des cabanes. (...) Beaucoup plus tard, ils retraceraient, nostalgiques, les contours de la maison d'enfance. (Chaix, Les Lauriers du lac de Constance, 1974)

(14) Sa maman était là, qui le serrera longuement dans ses bras à la fin. (...) Vendredi 19 février, pour la première fois depuis l'éclatement de son «affaire», Tiger Woods parlait publiquement. (Début de l'article « L'expiation publique calibrée du golfeur Tiger Woods », Le Monde, 22. 2. 2010)

En (11), la progression temporelle entre avoir lieu et faire renoncer est signifiée comme ultériorité objective par allait + inf. ; en (12), celle entre mettre à la voile et être rois, être reine, l'est par devai(en)t + inf.; en (13), celle entre chasser et regretter, retracer, l'est par le conditionnel (et le circonstant beaucoup plus tard); en (14), celle entre être là et serrer dans ses bras, l'est par le futur (et le circonstant à la fin).

Explicitons ce que nous entendons par ultériorité objective. Pour cela, revenons à l'emploi de base, à savoir l'ultériorité subjective, des tours :

(6) Alors il (Paul) eut une envie immense de fuir (...) loin de cette passion furieuse qui le ravageait. Il allait retourner à Chatou, prendre le train, et ne reviendrait plus, ne la reverrait plus jamais. (Maupassant, La Femme de Paul)

(7) c'était le mois prochain qu'ils devaient s'enfuir. Elle partirait d'Yonville comme pour aller faire des commissions à Rouen. (Flaubert, Mme Bovary)

Les procès, calculés comme ultérieurs à partir de l'acte d'énonciation de Paul (6), d'Emma (7), ne sont pas inscrits dans l'irrévocable du PASSÉ. Les formes périphrastiques allait /devait + inf., pas plus que le conditionnel, ne disent si les actes de retourner, ne pas revenir, ne plus revoir en (6); de s'enfuir et de partir en (7) se sont réalisés ou non. Le contexte ultérieur du récit nous apprendra qu'ils ne sont pas advenus au temps effectivement. Dans ces occurrences, on ne saurait, en équivalence sémantique grossière, remplacer ces formes par le passé simple $(6 b)$; on peut par contre ajouter des adverbes épistémiques (peut-être, etc.) (6c) :

(6b) Alors il eut une envie immense de fuir (...) Il allait retourner à Chatou, prendre le train, et ne reviendrait plus, ne la reverrait plus jamais $\neq \mathrm{Il}$ retourna à Chatou, prit le train, et ne revint plus, ne la revit plus jamais.

(6c) Il allait probablement retourner à Chatou, prendre le train, et ne reviendrait peut-être plus, ne la reverrait certainement plus jamais.

Les choses sont bien différentes avec les cas d'ultériorité objective de (11)-(14). Les procès allait faire renoncer (11), devaient être rois (12), regretteraient et retraceraient (13), serrera (14), sont posés comme ultérieurs à partir non d'une subjectivité, mais du précédent procès (respectivement : eut lieu, mit la voile, chassa, était). Ils sont inscrits dans l'irrévocable du PASSÉ, à savoir qu'ils sont signifiés comme s'étant effectivement réalisés, ce que teste leur possible remplacement, à une nuance expressive près, par le passé simple (13a) (ou, suivant le cotexte, par le passé composé ou l'imparfait); comme l'impossible ajout d'un adverbe comme peut-être (13b) :

(13a) L'été 43 chassa la petite famille de la maison aux toits d'ardoise. Beaucoup plus tard, les enfants regrettèrent les cerisiers, les buissons drus où ils enfouissaient des cabanes. (...)

(13b) ??Beaucoup plus tard, les enfants regretteraient peut-être les cerisiers, les buissons drus où ils enfouissaient des cabanes.

On dira en conséquence que allait/devait + inf., le conditionnel, ainsi que le futur, participent à la production, en (11) - (14), d'un effet de sens d'ultériorité objective.

Avant même d'analyser cette innovation, notons qu'elle est relativement récente : elle date de la période moderne $\left(\mathrm{XVIII}^{\mathrm{e}}-\mathrm{XIX}^{\mathrm{e}}\right)$, et s'est réalisée avec plus ou moins de précocité selon le morphème : dès le 
$\mathrm{XVIII}^{\mathrm{e}} \mathrm{s}$, pour le futur (en cotexte de présent historique), ainsi que pour allait / devait + inf. ; au XIX ${ }^{\mathrm{e}} \mathrm{s}$, pour le conditionnel :

(15) Il exclut également celui qui deviendra roi de France et celui qui deviendra empereur, ne voulant pas que le royaume d'Espagne soit possédé par aucune de ces puissances. (Dangeau, Journal, 1700)

(16) Le fils aîné commençait à donner des marques de ce qu'il devait être un jour; mais ce précieux enfant n'avait pas de santé et, à l'âge de douze ans, il fallut lui faire la plus cruelle des opérations. (Rétif de la Bretonne, La Vie de mon père, 1778)

(17) C'était encore une manière de mortifier le roi d'Angleterre, en assurant le Meklembourg à Pierre, déjà maître de la Livonie, et qui allait devenir plus puissant en Allemagne qu'aucun électeur. (Voltaire, Histoire de l'Empire de Russie sous Pierre le Grand, 1763)

(18) Ainsi liée à une doctrine qui ne pouvait longtemps rester progressive, la morale devait ensuite se trouver de plus en plus affectée par le discrédit croissant qu'allait nécessairement subir une théologie qui, désormais rétrograde, deviendrait enfin radicalement antipathique à la raison moderne. (Comte, Discours sur l'esprit positif, 1844)

Notre hypothèse est que cette innovation s'est faite par grammaticalisation des éléments subjectifs à la base de la structure des formes périphrastiques allait/ devait + inf., et des formes synthétiques du conditionnel et du futur. Dans le cadre de cet article, nous ne développerons que la grammaticalisation affectant le conditionnel.

\section{Le conditionnel : de l'ultériorité subjective à l'ultériorité objective}

Dans les tours (13) et (18), le conditionnel objectif se distingue donc du conditionnel subjectif en ce qu'il peut être remplacé par un passé simple et n'admet pas sa modalisation par un adverbe épistémique. Ajoutons une autre différence : alors que nous avons vu qu'un procès au conditionnel subjectif peut, en fonction du co(n)texte, avoir sa référence dans le PASSÉ, le PRÉSENT ou le FUTUR, l'emploi objectif s'inscrivant dans l'irrévocable, la référence du procès ne peut relever que du PASSÉ. Le procès n'admet pas de circonstant de simultanéité ou d'ultériorité au nunc:

(13b) L'été 43 chassa la petite famille de la maison aux toits d'ardoise. Les enfants regretteraient les cerisiers *aujourd'hui / *dans les prochaines années

L'emploi objectif, que décrivirent, les premiers, Damourette et Pichon (1911-1936, § 1842), questionne : par quel chemin une forme qui au départ signifie l'ultériorité subjective ${ }^{14}$ peut-elle en arriver à entrer dans la production d'un énoncé dans lequel le procès apparaît bien comme ultérieur mais objectivement; s'inscrit non dans la ramification mais dans l'unilinéarité de l'irrévocable ; et implique non la suspension du jugement épistémique du locuteur, mais, par inférence, l'engagement dans la factualité ?

Partons de la valeur de base du conditionnel : nous avons vu que son emploi implique une énonciation rapportée (e) située dans le PASSÉ (affixe -ai) de (E) et la construction du procès comme anaphoriquement ultérieur à l'énonciation (e) (affixe $-r$-), ce qui, par inférence inscrit le procès dans la ramification des possibles. On peut réécrire ces deux éléments de base de la structure du conditionnel subjectif sous la forme de quatre ingrédients :

a) contexte passé + b) énonciation rapportée $(e)+c)$ procès ultérieur + d) inférence : ramification des possibles

On pourrait penser que le conditionnel objectif consiste en un effacement des ingrédients b) (énonciation (e)) et d) (inférence de la ramification), pour ne garder que les éléments a) et c). Soit donc :

a) contexte passé + b) énonciation rapportée $(e)+c)$ procès ultérieur $\neq d$ ) inférence : ramification des pessibles

Et effectivement, dans : 
(13) L'été 43 chassa la petite famille de la maison aux toits d'ardoise. Beaucoup plus tard, les enfants regretteraient les cerisiers (...)

le conditionnel semble simplement placer, dans le PASSÉ, en appui sur le circonstant beaucoup plus tard, le procès regretter comme ultérieur au procès précédent.

Ce serait cependant une approximation simplificatrice. Nous ferons plutôt l'hypothèse que s'est réalisé un travail d'extension contextuelle sur l'élément b) (énonciation (e)) qui a conduit à son extrême dilution mais pas à sa disparition, dans la mesure où il persiste sous forme de traces dans l'effet de sens produit de mise en perspective. Ce travail d'extension, nous proposons d'en voir les premières manifestations dans deux types de structures syntaxiques qui apparaissent au XVIII ${ }^{\mathrm{e}} \mathrm{s}$ : (i) l'affaiblissement de l'énonciation rapportée (e); (ii) la négation ou l'interrogation de l'énonciation (e).

(i) Prototypiquement, le conditionnel subjectif se trouve dans des complétives ou des interrogatives indirectes dont la principale explicite l'énonciation rapportée (e) :

(20) L'Espagne se flattait alors que le Japon deviendrait une de ses provinces, et le Saint Siège voyait déjà le tiers de cet empire soumis à sa juridiction ecclésiastique. (Voltaire, Essay sur l'histoire générale et sur les moeurs et sur l'esprit des nations, 1756)

Le procès deviendrait est posé comme ultérieur, s'originant dans la subjectivité de l'actant Le Japon et dans son acte d'énonciation se flattait.

On trouve à partir du XVIII ${ }^{\mathrm{e}} \mathrm{s}$, notamment dans les ouvrages historiques de Voltaire, des occurrences de conditionnel en subordonnées circonstancielles (ou relatives), dans lesquelles l'énonciation rapportée n'est plus que présupposée, voire sous-entendue :

(21) Villelongue se chargea de remettre lui-même ce paquet entre les mains du Grand Seigneur, lorsqu'il irait à la mosquée, selon la coutume. (Voltaire, Charles XII, apud Nillson-Ehle, op. cit., 60)

(22) Charles, à la tête de sa cavalerie, fit trente lieues en vingt-quatre heures, chaque cavalier menant un cheval en main pour le monter quand le sien serait rendu.

(Voltaire, Charles XII, apud Nillson-Ehle, op. cit., 61)

Dans cette structure syntaxique de circonstancielle : « lorsqu'il irait à la mosquée », " quand le sien serait rendu », le procès au conditionnel n'est plus dans la perspective directe d'un verbe de pensée ou de parole. Il est cependant interprété comme procédant indirectement d'un acte d'énonciation : en (21), se chargea implique un engagement verbal de l'actant Villelongue; en (22), le circonstant de but «pour le monter » sous-entend la subjectivité de « chaque cavalier» anticipant sur la fatigue de son cheval.

Ce type de contexte qui affaiblit la présence de l'énonciation (e) nous semble préparer les tours où cet élément ne sera plus présent que sous forme de traces dans l'effet de sens produit.

(ii) Tout aussi et peut-être plus important pour l'évolution de l'ultériorité subjective en ultériorité objective, fleurit à partir des $\mathrm{XVIII}^{\mathrm{e}} \mathrm{s}$ et $\mathrm{XIX}^{\mathrm{e}} \mathrm{s}$ un tour qui présente bien une énonciation explicite (e), mais à la modalité négative ou interrogative (partielle rhétorique) :

(23) Cependant il (Pierre Le Grand) était récemment marié ; mais (...) les liens sérieux du mariage ne le retinrent pas assez. Les plaisirs de la table avec quelques étrangers attirés à Moscou par le ministre Galitzin, ne firent pas augurer qu'il serait un réformateur ; cependant malgré les mauvais exemples, et même malgré les plaisirs il s'appliquait à l'art militaire et au gouvernement : on devait déjà en lui reconnaître le germe d'un grand homme. On s'attendait encore moins qu'un prince qui était saisi d'un effroi machinal qui allait jusqu'à la sueur froide et à des convulsions quand il fallait passer un ruisseau, deviendrait un jour le meilleur homme de mer dans le septentrion. Il commença par dompter la nature en se jetant dans l'eau malgré son horreur pour cet élément ; l'aversion se changea même en un goût dominant. (Voltaire, Histoire de l'Empire de Russie sous Pierre le Grand, 1759) 
(24) Le piquant, c'est qu'elle me fut en quelque sorte donnée par ceux qui la voulaient pour eux. L'un lui apporta mon livre et l'entretint de mes cours. L'autre (...) se vanta auprès d'elle de ses rapports avec moi et vanta mon caractère. Ni l'un, ni l'autre ne supposaient qu'ils travaillaient pour moi et qu'elle deviendrait mienne. (Michelet, Journal, 1848)

(25) Hélas ! Qui dans ce temps aurait pu croire qu'un tel homme serait un jour accusé d'être dur, arrogant et factieux? Ah! Jamais une âme plus pure n'a traversé la région des orages! (Staël de, Considérations sur les principaux évènements de la Révolution française, 1817)

Comme (20) supra, ces occurrences réalisent le conditionnel en complétive, qui construit l'ultériorité à partir d'une énonciation subjective explicite.

Mais la négation $(23,24)$, la comparaison d'infériorité en (23), ou l'interrogation rhétorique en (25), qui frappent le verbe introducteur de parole ou de pensée, frappent du même coup son objet, à savoir la complétive. Or infirmer que les procès être un réformateur, devenir un homme de mer, devenir mienne, être accusé, puissent avoir fait l'objet d'une énonciation qui les pose comme ultérieurement possibles, induit, par inférence, que Pierre Le Grand a été effectivement un réformateur, qu'il est devenu effectivement un homme de mer (23); qu'elle est devenue effectivement mienne (24), qu'un tel homme a effectivement été accusé (25). La présence de cette inférence peut être explicitée par une relative qui reprend anaphoriquement cet élément de sens latent (24a), alors que sa négation produit un énoncé douteux $(24 b)$ :

(24a) Le piquant, c'est qu'elle me fut en quelque sorte donnée par ceux qui la voulaient pour eux. (...) Ni l'un, ni l'autre ne supposaient (...) qu'elle deviendrait mienne. Ce qu'elle fut.

(24b) Le piquant, c'est qu'elle me fut en quelque sorte donnée par ceux qui la voulaient pour eux. (...) Ni l'un, ni l'autre ne supposaient (...) qu'elle deviendrait mienne. ??Ce qu'elle ne fut pas.

Soulignons que ces faits objectifs ne sont pas posés, ce qui explique qu'on ne puisse pas dans ces tours remplacer le conditionnel par un temps du PASSÉ effectif, ici l'imparfait :

(25a) *Qui dans ce temps aurait pu croire qu'un tel homme était un jour accusé d'être dur, arrogant et factieux?

Le conditionnel n'a donc pas cessé d'être subjectif : simplement, soumis à une énonciation rapportée (e) négative, il laisse entendre que le procès qui n'a pas fait l'objet d'une énonciation subjective correspond à la réalité objective des faits ultérieurs.

Les deux types de contexte que nous venons d'analyser - l'affaiblissement de l'énonciation (e), son insertion dans un énoncé négatif ou interrogatif - qui apparaissent et se répandent à partir du XVIII ${ }^{\mathrm{e}} \mathrm{s}$, sans sortir de l'expression de l'ultériorité subjective, laissent affleurer la possibilité de l'expression de l'ultériorité objective, qu'ils nous semblent préparer.

Ce sera chose faite au cours de la première moitié du XIX ${ }^{\mathrm{e}}$. Proposons, outre (18) supra, (26) :

(26) Mais, à son insu, l'égoïsme lui avait été inoculé. Les germes de l'économie politique à l'usage du parisien, latents en son cœur, ne devaient pas tarder à y fleurir, aussitôt que de spectateur oisif il deviendrait acteur dans le drame de la vie réelle. (Balzac, Eugénie Grandet, 1833) $^{15}$

Ce qui n'était qu'une inférence dans les exemples (23) - (25) devient ici un élément de sens nouveau actualisable par le conditionnel : l'inscription du procès dans la factualité unilinéaire du PASSÉ. Le possible remplacement par un passé simple vérifie qu'on a bien affaire à une ultériorité objective :

(26a) Les germes de l'économie politique à l'usage du parisien, latents en son cœur, ne tardèrent pas à y fleurir, aussitôt que de spectateur oisif il devint acteur dans le drame de la vie réelle. 
Il nous semble intéressant de noter que, dans ces occurrences, le conditionnel apparaît après une ou deux formes périphrastiques elles-mêmes objectives :

$$
\begin{aligned}
& \text { (18) devait se trouver }>\text { allait subir }>\text { deviendrait } \\
& \text { (26) devaient pas tarder }>\text { deviendrait }
\end{aligned}
$$

comme s'il en était une variante stylistique, encore peu assurée dans son nouveau fonctionnement objectif, et qui a besoin d'être préparée en quelque sorte par les tours périphrastiques plus avancés puisque datant du siècle précédent - dans l'expression de l'ultériorité objective.

Qu'est-il advenu de l'énonciation de (e) qui signait la subjectivité de l'ultériorité ? Purement et simplement disparue ? On pourrait le penser : si on prend p.e. (26), le fait que le personnage devienne acteur est présenté comme un fait s'inscrivant dans l'unilinéarité du passé, hors médiation subjective de tout actant.

Reprenons la formule par laquelle on a défini le conditionnel subjectif :

a) contexte passé + b) énonciation enchâssée $(e)+c)$ procès ultérieur $+d)$ inférence : ramification des possibles

et analysons plus précisément (13) :

(13) L'été 43 chassa la petite famille de la maison aux toits d'ardoise. Beaucoup plus tard, les enfants regretteraient les cerisiers, les buissons drus où ils enfouissaient des cabanes. (...) Beaucoup plus tard, ils retraceraient, nostalgiques, les contours de la maison d'enfance. (Chaix, Les Lauriers du lac de Constance, 1974)

a) contexte : la narratrice raconte sa petite enfance de fille de collaborateur notoire, sans sortir du PASSÉ ;

c) procès ultérieur : le circonstant beaucoup plus tard, répété devant chaque occurrence, place les procès regretter et retracer comme ultérieurs.

b) Mais ultérieurs par rapport à quoi ? Assurément pas par rapport à une énonciation rapportée (e) : les procès au conditionnel sont dans des propositions syntaxiquement indépendantes, et prolongent la narration objective de la phrase précédente au passé simple : on ne saurait y voir du discours indirect libre procédant de la pensée de «la petite famille». L'énonciation (e) n'est pas effacée mais s'est grammaticalisée : c'est grâce à sa persistance, sous une forme très abstraite, qu'est produit l'effet de sens de mise en perspective que les différents grammairiens s'accordent à attribuer à ce tour et qui différencie l'emploi du conditionnel de celui du passé simple. Si regretter et retracer étaient actualisés au passé simple :

(13a) L'été 43 chassa la petite famille de la maison aux toits d'ardoise. Beaucoup plus tard, les enfants regrettèrent les cerisiers, les buissons drus où ils enfouissaient des cabanes. Beaucoup plus tard, ils retracèrent, nostalgiques, les contours de la maison d'enfance.

ce temps s'accorderait à la relation de progression produite cotextuellement : il n'en serait en rien un ingrédient actif. Ce qui n'est pas le cas du conditionnel en (13) : de par sa structure de placement du procès en ultériorité par rapport à un repère énonciatif passé, ce temps participe activement à la relation de progression dans la mesure où il demande de concevoir regretter et retracer comme ultérieurs par rapport à un point de vue passé - celui sur lequel le narrateur propose au narrataire de s'aligner, et qui temporellement s'adosse au précédent procès (chassa). Ce point de vue, que nous analysons comme la grammaticalisation par abstraction de l'énonciation (e), n'a pas de corps - il ne correspond pas à un actant précis doté d'un acte d'énonciation explicite ou implicite comme dans le discours rapporté - autre que celui que lui confère le jeu de l'énonciation narrative elle-même.

d) Qu'advient-il de l'élément d), l'inférence selon laquelle l'ultériorité implique la ramification des possibles ? Les différentes branches ouvertes par le conditionnel se voient aussitôt émondées par le contexte, qui ne conserve que celle de la réalisation effective. 
Ce qui est objectif, ce n'est donc pas le conditionnel mais l'effet de sens résultant de l'interaction partiellement discordante entre un contexte passé qui demande que le procès soit actualisé comme factuel (au passé simple notamment), et un temps verbal, le conditionnel qui, sans cesser d'être lui-même, grammaticalise certains de ses composants - très précisément l'énonciation rapportée (e) - à savoir non pas les efface mais les rend plus abstraits pour qu'ils soient compatibles avec le contexte d'accueil. Le rejeton de cette interaction quelque peu dissonante, c'est l'effet de style de mise en perspective qui fait que ce type de tour apparaît comme fortement marqué.

Le chemin de grammaticalisation qui conduit le conditionnel de l'ultériorité subjective à l'ultériorité objective n'a rien d'une pente douce, ce dont nous semblent témoigner les trois faits suivants :

- la longueur dudit chemin : plus de dix siècles; et dans cette course au long cours, le conditionnel fut précédé par les périphrases modale (devait + inf.) et temporelle (allait + inf.), ainsi que par le futur dit historique $\left(\mathrm{XVIII}^{\mathrm{e}}\right)$ : c'est que ces structures parviennent à signifier l'ultériorité objective plus facilement, dans la mesure où leur interaction avec un contexte d'irrévocabilité passée est moins dissonante. Ce chemin de grammaticalisation n'est pourtant pas une exception française : d'autres langues romanes, notamment le catalan (28) et l'espagnol (29), l'ont également parcouru, et avec aussi peu de rapidité :

(28) Es la tradicio que Sarkozy també va elogiar. Després aniria caminant cap al discurs public. I tornaria a tocar mans. I a assentir quan Bartumeu va recordar que s'ha deixat la llista grisa dels paradisos. (Diari d'Andorra, 30 juillet 2010. Visite de Sarkozy en Andorre) ('Sarkozy a fait l'éloge de la tradition. Ensuite il se dirigerait en marchant vers le lieu où il devait faire un discours en public. Et il serrerait à nouveau des mains. Et approuverait lorsque Bartumeu a rappelé qu'Andorre est sorti de la liste grise des paradis')

(29) La huelga y la insurección de esa Semana Trágica dejó un saldo de 104 paisanos muertos y ocho guardias heridos. Hubo alrededor de 2000 detenidos, de los cuales 600 serían condenados, 59 a cadena perpetua y 17 a muerte, auque sólo se ejecutó a cinco. (El Pais, 11 août 2009 ; article Opinión) ('la grève et l'insurrection de cette semaine tragique se soldèrent par 104 morts dans la population et 8 gardiens de l'ordre blessés. Il y eut environ 2000 personnes arrêtées, parmi lesquelles 600 seraient condamnées, 59 à la prison à vie et 17 à mort, bien que seulement 5 furent exécutées')

- l'usage encore limité du conditionnel objectif : on le trouve dans différents genres du discours (histoire, compte-rendu sportif, nécrologie, roman, etc.) de la textualité narrative écrite, mais toujours sporadiquement (à quelques exceptions près, comme dans le roman Nous trois de J. Echenoz); et il n'a pas pénétré l'oral des interactions verbales, pas plus que les genres textuels familiers de l'écrit électronique.

- Plus d'un siècle après sa naissance, il fait encore l'objet de commentaires métalinguistiques d'ostracisme : p.e. pour R. Martin, cet emploi «paraît si contraire à la nature véritable de ce temps grammatical qu'il en est comme déformé et grimaçant, et qu'on le reconnaît à peine. (...) Il n'a guère de chance de s'imposer : il paraîtra toujours incorrect et, à tout prendre, maladroit » $(1971: 125)$.

\section{Conclusion}

Le français, comme d'autres langues romanes, s'est doté, dès l'origine, de moyens pour signifier l'ultériorité subjective dans le PASSÉ (formes périphrastiques allait/ devait + inf., conditionnel), sur le modèle de l'ultériorité du PRÉSENT.

Il a réussi ensuite à développer, par grammaticalisation de l'élément subjectif, l'expression de l'ultériorité objective dans le PASSÉ, par un cheminement que nous avons esquissé pour le conditionnel.

La langue a su grammaticaliser périphrastiquement (auxiliaires : devait/allait + inf.) et morphologiquement (futur, conditionnel) sur le verbe un élément qui ne pouvait jusqu'au XVIII's se signifier que contextuellement: l'ultériorité objective dans le PASSÉ. On ne peut qu'admirer ce tour de force qui consiste à introduire de la perspective dans l'unilinéarité irrévocable du PASSÉ. 
On remarquera enfin que le développement d'un effet de sens objectif au conditionnel qui s'est opéré par la grammaticalisation de la médiation énonciative au principe de cette forme est allé dans le sens inverse des emplois dialogiques du futur (cf. supra, occurrence (5) de la note 6) et de l'imparfait qui s'obtiennent par dégagement d'une énonciation intermédiaire (Bres, 2009). La langue serait-elle une magicienne qui tantôt fait disparaître tantôt fait apparaître une strate énonciative ? Cette question engage à revenir sur le rapport plus général du temps verbal avec la subjectivité, qu'elle soit de l'ordre de l'énonciation ou de l'ordre du point de vue (cf. note 11 ; et également (b) supra). Ce sera l'objet d'un prochain travail.

\section{Bibliographie}

Azzopardi, S. (2011). Le futur et le conditionnel : valeur en langue et effets de sens en discours. Analyse contrastive espagnol / français. Thèse de doctorat, Université de Montpellier 3,http://www.biumontpellier.fr/florabium/jsp/nnt.jsp?nnt=2011MON30052.

Benveniste, E. (1959/1966). Les relations de temps dans le verbe français, in Problèmes de linguistique générale. Paris : Gallimard, 237-257.

Bres, J. (2003). Temps verbal, aspect et point de vue : de la langue au discours. Cahiers de praxématique 41, 55-84.

Bres, J. (2009). Dialogisme et temps verbaux de l'indicatif. Langue Française 163, 21-39.

Bres, J. (2010). Arthur, où t'as mis ton corps ? Conditionnel et actualisation de l'effet de sens historique, in Bres, J., Arabyan, M., Ponchon, Th., Rosier, L., Tremblay, R., et Vachon-l'Heureux, P., Actes du colloque international de l'AIPL, Le concept d'actualisation en psychomécanique, juin, Bruxelles, Limoges : Lambert Lucas, 193-204.

Bres, J. et Labeau, E. (2012, à par.). Un phénix linguistique ? Le tour narratif va+infinitif renaîtrait-il, en français contemporain, de ses cendres médiévales?, Actes du colloque Diacro V, octobre 2010, Lyon.

Bybee, J., Perkins, R. et Pagliuca, W. (1994). The evolution of grammar. Tense, aspect and modality in the languages of the world, Chicago : University of Chicago Press.

Damourette, J. \& Pichon, J. (1911-1936). Des mots à la pensée: Essai de grammaire de la langue française (Tome 5). Paris : D'Artrey.

Hagège, Cl. (1993). The language builder. Amsterdam : John Benjamins.

Gardies, J.-L. (1975). La logique du temps. Paris : PUF.

Martin, R. (1981). Le futur linguistique : temps linéaire ou temps ramifié ? (à propos du futur et du conditionnel en français). Langages 64, 81-92.

Martin, R. (1971). Temps et aspect. Essai sur l'emploi des temps narratifs en moyen français. Paris : Klincksieck.

Nillson-Ehle, H. (1943). Le conditionnel « futur du passé » et la périphrase devait+infinitif, Studia neophilologica 16, 50-88.

Reichenbach, H. (1947). Elements of symbolic logic. New York: Free Press.

Saussure (de), L. et Sthioul, B. (1999). L'imparfait narratif: point de vue (et images du monde). Cahiers de praxématique $32,167-188$.

Sthioul, B. (1998). Temps verbaux et points de vue, in Moeschler, J. et al. (éds), Le temps des événements. Paris : Kimé, 197-220.

Sthioul, B. (2000). Passé simple, imparfait et sujet de conscience. Cahiers Chronos 6, 79-93.

Tournadre, N. (2004). Typologie des aspects verbaux et intégration à une théorie du TAM. Bulletin de la société de linguistique de Paris, XCIX, 1, 7-68.

Vuillaume, M. (2001). L'expression du futur du passé en français et en allemand, in Dendale, P. et Tasmowski, L. (éds), Le conditionnel en français. Paris, Klincsieck, 105-124.

\footnotetext{
${ }^{1}$ Afin de distinguer les époques passée, présente et future des temps verbaux, nous utilisons les majuscules pour signifier les premières.
} 


\begin{abstract}
${ }^{2}$ De fait, il faudrait dire de façon plus précise : comment signifier que le point de saisie aspectuelle d'un procès B, est, dans le PASSÉ, ultérieur au point de saisie d'un procès A ? Mais dans la mesure où, dans la présente analyse, la distinction procès/point de saisie n'a pas d'incidence, nous garderons la première formulation, pour sa simplicité, malgré son caractère approximatif.

${ }^{3}$ Précisons que nous ne prendrons en compte dans cette étude que les formes simples (à l'exception du passé composé, mais ici seulement considéré dans sa dimension d'aoriste du discours (Benveniste, 1959/1966: 249), c'est-à-dire équivalant, par son fonctionnement, à une forme simple, ce que signale notamment le fait qu'il est pourvu d'une forme composée, le passé surcomposé : Quand Corinne a eu mangé, elle est sortie). Il ne sera donc pas traité du futur antérieur, qui pose des problèmes spécifiques que nous ne pouvons aborder dans le cadre de cet article.
\end{abstract}

${ }^{4}$ Nous entendons par énonciation 'acte d'énonciation', qui implique différents paramètres (personnels, temporels, spatiaux, modaux). Cet acte se signifie linguistiquement notamment par un verbe de parole ou de pensée, qui peut être explicite ou implicite : (je dis que) Corinne est venue.

${ }^{5}$ Nous ne traitons ici que de l'emploi temporel de cette forme.

${ }^{6}$ Nous ne prenons pas en compte ici les emplois dialogiques où le futur réfère à un procès situé dans le PRÉSENT, comme p.e. dans le tour conjectural : (5) - Françoise, mais pour qui donc a-t-on sonné la cloche des morts ? Ah ! mon dieu ce sera pour Mme Rousseau. Voilà-t-il pas que j'avais oublié qu'elle a passé l'autre nuit. (Proust, A la recherche du temps perdu). Cf., i. a., Azzopardi 2011, chapitre 9 ; Bres, 2009.

${ }^{7} \mathrm{E}_{1}$ et $\mathrm{e}_{1}$ peuvent être en relation de coréférence (je disais que Corinne reviendrait) ou non (ex. (3)).

${ }^{8}$ Reichenbach (1947) explicite la structure du conditionnel en tant que posterior past par les trois formules : R-E-S ; R-E,S ; R-S-E.

${ }^{9}$ Aujourd'hui présuppose ici une ultériorité.

10 Cf. les incongruités de sémantique temporelle dans la poésie contemporaine: "A la poste d'hier tu télégraphieras/que nous sommes bien morts avec les hirondelles »(Desnos, Les Gorges froides).

${ }^{11}$ Entendons par là que la référence temporelle s'effectue via la médiation d'un acte d'énonciation. Nous rejoignons, mais dans un cadre différent, les propositions de Nillson-Ehle (1943). La subjectivité dont il est question ici est d'ordre énonciatif (qui parle?) ; son marquage est à distinguer de celui qui procède d'un point de vue (qui voit ?). Sur la question du rapport entre temps verbaux et point de vue subjectif, voir, dans les cadres de la théorie de la pertinence, Sthioul (1998 et 2000), Saussure \& Sthioul (1999); dans les cadres de la praxématique, Bres (2003).

${ }^{12}$ Nous ne parlerons pas ici du tour périphrastique ( $v a+$ inf.) qui peut également signifier l'ultériorité objective, mais est soumis à certaines contraintes cotextuelles spécifiques (cf. Bres et Labeau, 2012).

${ }^{13}$ Bientôt est facultatif, même si son absence fait sentir comme une ellipse : (8a) Elle souffla la bougie ; et tout le monde dormit aux deux étages de la maison. L'absence de l'adverbe semble plus naturelle avec l'inchoatif s'endormir : (8b) Elle souffla la bougie ; et tout le monde s'endormit aux deux étages de la maison.

${ }^{14}$ Cf. notamment la première occurrence datée, dans laquelle le conditionnel est subjectif (emploi que l'on dirait aujourd'hui de discours indirect libre): (19) Ell'ent aduret lo suon element: / Melz sostendreiet les empedementz/Qu'elle perdesse sa virginitet ('Eulalie se raidit: elle souffrirait la torture plutôt que de perdre sa virginité'). (Cantilène de Sainte-Eulalie, v. 16, 880).

${ }^{15}$ A. Patard a trouvé dans le texte de Rabelais, Gargantua, au chapitre XXXXVI, ce qui est peut-être la première occurrence de conditionnel objectif: (27) Les troys aultres le suivirent sans faillir, excepté Eudemon, duquel le cheval enfoncea le pied droict jusques au genoil dedans la pance d'un gros et vilain gras vilain qui estoit là noyé, à l'envers, et ne le povoit tirer hors; ainsi demoureroit empestré jusques à ce que Gargantua du bout de son baston enfonrda le reste des tripes du vilain en l'eau, ce pendant que le cheval levoit le pied. Ce conditionnel, qui apparaît dans la première version (1535), sera remplacé, dans la dernière (1542), par un imparfait : demeuroit. Je remercie A. Steuckardt qui m'a fourni cette précision. Peut-on interpréter cette correction comme la marque de ce que Rabelais eut conscience que le conditionnel, dans cette occurrence, outrepassait la sémantique grammaticale de la langue? 